clamping. However, their cardiomegaly and reduced ejection fractions suggested difficulty in approaching targets on the lateral and inferior walls without hemodynamic compromise.

Several techniques have been developed to minimize the hemodynamic changes during $\mathrm{OPCAB}$, including volume loading, inotropes, patient positioning, deep pericardial retraction sutures, right pleurectomy, right hemisternal elevation, pulmonary artery shunts, intracoronary shunts, and cardiac assist devices. ${ }^{1-5}$ Even with full use of these techniques, however, exposure of lateral wall targets through a median sternotomy may not be possible for certain patients with severe cardiomegaly or poor function. The LATS incision was used in this group of patients because of the perception that it would allow the full range of surgical options for OPCAB, with less cardiac manipulation and hopefully less hemodynamic disruption than a midline incision. This is because, unlike a median sternotomy, a LATS incision is centered near the cardiac apex. Thus less rotation is needed to view the lateral and inferior walls, and there is no left sternal edge to obstruct the surgeon's view. In addition, the heart is not compressed against the sternum when rotated to the right.

The thoracosternal incision is associated with poor wound healing and an elevated risk of sternal nonunion. Also, rib-spreading incisions are notorious for causing postoperative pain. Fortunately, we did not observe any significant incision-related difficulties in our small series. However, more experience will be required before thoracosternotomy could be considered for broader application.

In conclusion, a LATS incision allows excellent exposure of all target vessels, with no significant compromises in routine operative technique. It should be considered in situations where multivessel OPCAB is indicated but the requisite cardiac displacement may be poorly tolerated.

\section{References}

1. Koutlas TC, Elbeery JR, Williams JM, Moran JF, Francalancia NA, Chitwood WR Jr. Myocardial revascularization in the elderly using beating heart coronary artery bypass surgery. Ann Thorac Surg. 2000; 69:1042-7.

2. Bergsland J, Hasnan S, Lewin AN, Bhayana J, Lojos TZ, Salerno TA. Coronary bypass grafting without cardiopulmonary bypass-an attractive alternative in high risk patients. Eur J Cardiothorac Surg. 1997; 11:876-80.

3. Mathison M, Edgerton JR, Horswell JL, Akin JJ, Mack MJ. Analysis of hemodynamic changes during beating heart surgical procedures. Ann Thorac Surg. 2000;70:1355-61.

4. Porat E, Sharony R, Ivry S, Ozaki S, Meyns BP, Flameng WJ, et al. Hemodynamic changes and right heart support during vertical displacement of the beating heart. Ann Thorac Surg. 2000;69:1188-91.

5. Luchetti V, Capasso F, Caputo M, Grimaldi G, Capece M, Brando G, et al. Intracoronary shunt prevents left ventricular function impairment during beating heart coronary revascularization. Eur J Cardiothorac Surg. 1999;15:255-9.

\title{
Bilateral diaphragmatic paralysis after aortic surgery with topical hypothermia: Ventilatory assistance by means of nasal mask bilevel positive pressure
}

Yoshiyuki Tokuda, MD, Masahiko Matsumoto, MD, Takaaki Sugita, MD, Junichiro Nishizawa, MD, Katsuhiko Matsuyama, MD, Kazunori Yoshida, MD, and Takehiko Matsuo, MD, Nara, Japan

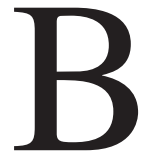

ilateral diaphragmatic paralysis is a rare but severe complication of cardiac surgery mainly caused by hypothermic injury of phrenic nerves. ${ }^{1,2}$ Most patients with bilateral diaphragmatic paralysis have been treated with positive-pressure ventilators through tracheostomy. ${ }^{3}$

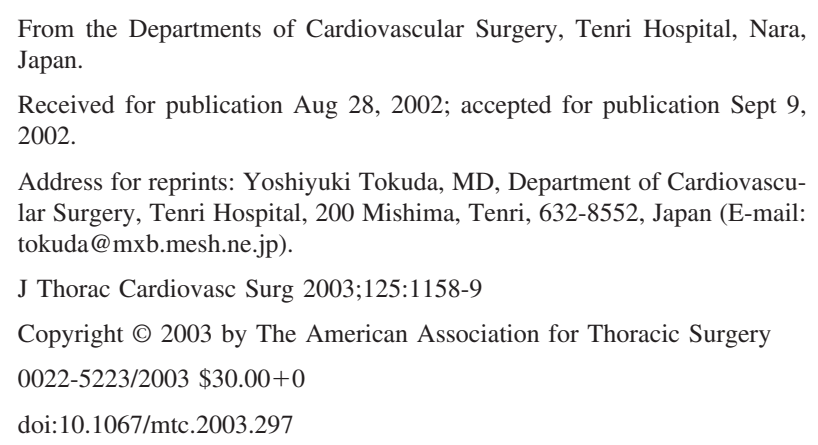

Recently, noninvasive positive airway ventilation has been applied to various types of respiratory failure. We report the case of a 35-year-old man who had postoperative bilateral diaphragmatic paralysis and was treated with nasal mask bilevel positive airway pressure (BiPAP) ventilation.

\section{Clinical Summary}

A 35-year-old man with a history of renal dysfunction and hypertension complained of sudden chest pain and was referred to our hospital. Computed tomography revealed the presence of Stanford type A acute aortic dissection and a large amount of pericardial effusion. The patient was in shock because of cardiac tamponade.

The patient underwent an emergency operation. During the operation, cardiopulmonary bypass was established with right atrial venous cannulation and left femoral arterial cannulation. The patient was cooled to $25^{\circ} \mathrm{C}$ by using the cardiopulmonary bypass circuit. The dilated ascending aorta was opened vertically, and antegrade selective cerebral perfusion was performed for all 3 arch vessels. For myocardial protection, St Thomas solution was di- 
rectly administered into the coronary arteries, and topical cooling of the heart with ice slush was performed. The aortic dissection ranged from the aortic root to the iliac bifurcation; however, the aortic valve, coronary orifices, and arch vessels were not involved with the dissection. The intimal tear was found in the aortic arch just below the brachiocephalic artery. The ascending aorta and proximal aortic arch were replaced with a woven Dacron graft by using the open anastomosis technique. The operative procedure was straightforward, and no apparent direct injury to the phrenic nerve occurred. The patient was easily weaned from cardiopulmonary bypass.

After the operation, the patient required hemodialysis for renal dysfunction. The patient also had liver dysfunction, probably as a result of preoperative shock status. After the improvement of these conditions, weaning from assisted ventilation was attempted. Weaning required 2 days, and the patient was extubated on the 10th postoperative day. After extubation, he was found to have tachypnea and then had respiratory failure. Chest radiography showed a bilaterally raised diaphragm. Fluoroscopic examination showed paradoxical movement of the bilateral diaphragm. The transcutaneous phrenic nerve stimulation test showed almost absent compound diaphragmatic motor action potential bilaterally, which confirmed bilateral diaphragmatic paralysis of the nerve (Figure 1). Therefore the patient was placed on nasal mask BiPAP ventilation to avoid repeated intubation or tracheostomy. The inspiratory support pressure was set at $10 \mathrm{~cm} \mathrm{H}_{2} \mathrm{O}$, and the expiratory pressure was set at $5 \mathrm{~cm} \mathrm{H}_{2} \mathrm{O}$ by using a BiPAP Vision (Respironics Inc) ventilatory support system. The ventilatory support was effective and well tolerated. The patient was then able to sleep in the supine position without breathing difficulties. The patient had only scanty sputum and was very cooperative during treatment.

After ventilatory assist with BiPAP for 4 months, the patient recovered spontaneously from the paralysis and was successfully weaned from BiPAP ventilation. After discharge, the patient currently leads an independent life without ventilatory assistance as an office worker.

\section{Discussion}

Hypothermic injury of the phrenic nerve is regarded as the main cause of postoperative diaphragmatic paralysis, although several other mechanisms have been proposed, such as direct surgical injury of the phrenic nerve and sternal retraction. ${ }^{1}$ Phrenic nerve dysfunction caused by hypothermic injury is usually reversible, but spontaneous recovery requires several months. Thus most patients with bilateral diaphragmatic paralysis have been treated with positive-pressure ventilators through a tracheostomy until recovery occurs. ${ }^{2,3}$

For the present patient, however, nasal mask BiPAP ventilation was chosen instead. It has been applied to various types of hypercapnic and hypoxic respiratory failure, and several published studies support its use. ${ }^{4}$ It improves patients' quality of life by avoiding intubation or tracheostomy and is thus of great benefit. On the other hand, one must consider the potential disadvantage of BiPAP ventilation. Nasal mask BiPAP ventilation requires a patient's cooperation during treatment, including self-excretion of sputum.
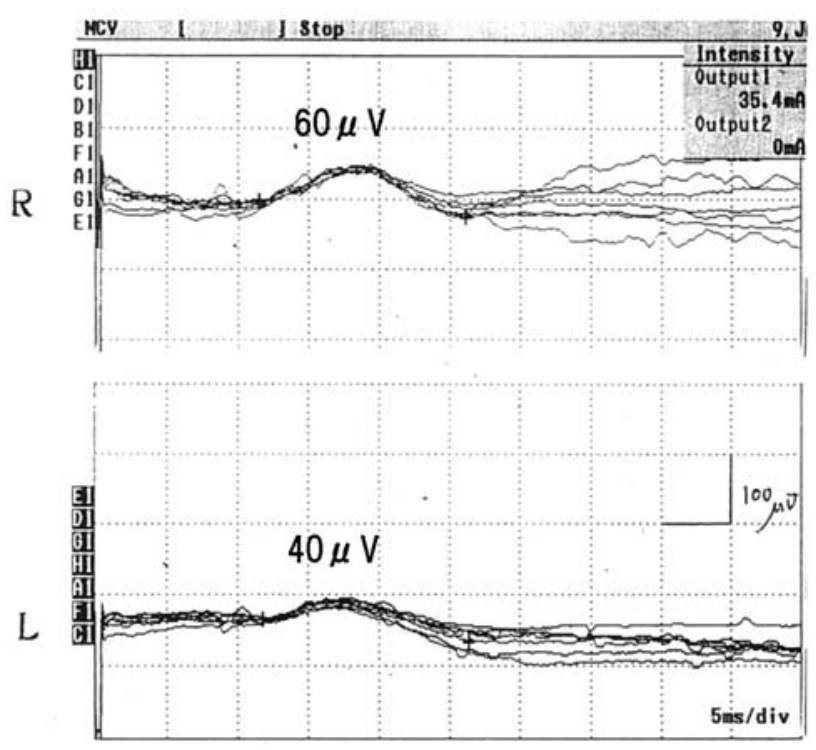

Figure 1. Phrenic nerve function was investigated by means of percutaneous phrenic nerve stimulation at the neck and recording of the compound diaphragmatic motor action potential at the lower chest wall. The stimulation evoked very-low-amplitude compound diaphragmatic motor action potential bilaterally (60 $\mu \mathrm{V}$ at right, $40 \mu \mathrm{V}$ at left; normal value, $>350 \mu \mathrm{V}$ ). ${ }^{2}$ During the stimulation, no twitch in the diaphragm was observed. These results confirmed bilateral diaphragmatic paralysis.

Furthermore, there are risks specific to BiPAP ventilation, such as failure to provide sufficient oxygenation and $\mathrm{CO}_{2}$ elimination, eye or nasal trauma, and gastric distention. Patient comfort, as well as cyanosis, tachypnea, and conventional vital signs, should be carefully monitored. In addition, because the existence of mask leaks is associated with a higher incidence of failure, close monitoring for leaks is mandatory. ${ }^{4}$

The benefits of BiPAP ventilation must be balanced against the disadvantages on a case-by-case basis. However, we recommend that nasal mask BiPAP ventilation be tried first, as opposed to direct tracheostomy, for postoperative bilateral diaphragmatic paralysis.

\section{References}

1. Mazzoni M, Solinas C, Sisillo E, Bortone F, Susini G. Intraoperative phrenic nerve monitoring in cardiac surgery. Chest. 1996;109:145560.

2. Efthimiou J, Butler J, Benson MK, Westaby S. Bilateral diaphragm paralysis after cardiac surgery with topical hypothermia. Thorax. 1991;46:351-4.

3. Brown KA, Hoffstein V, Byrick RJ. Bedside diagnosis of bilateral diaphragmatic paralysis in a ventilator-dependent patient after openheart surgery. Anesth Analg. 1985;64:1208-10.

4. International Consensus Conferences in Intensive Care Medicine. Noninvasive positive pressure ventilation in acute Respiratory failure. Am J Respir Crit Care Med. 2001;163:283-91. 